\title{
Ratings of perceived exertion in braille: validity and reliability in production mode
}

\author{
John P Buckley, Roger G Eston, Julius Sim
}

\begin{abstract}
Objectives- $(a)$ To assess the validity and reliability of producing and reproducing a given exercise intensity during cycle ergometry using a braille version of Borg's standard 6-20 rating of perceived exertion (RPE) scale, and (b) to determine whether the exercise responses of blind participants, at a given produced RPE, were similar to those reported in recognised guidelines for sighted subjects.
\end{abstract}

Methods-Ten healthy registered blind volunteer participants (four women, six men; mean (SD) age 23.2 (9.0) years) performed an initial graded exercise cycle test to determine maximal heart rate (HRMAx) and maximal oxygen uptake (Vo ${ }_{2}$ Max). Three trials of three exercise bouts at RPEs 9, 11, and 13 were then performed in random order on three separate days of the same week, with expired air and heart rate measured continuously. Each exercise bout was followed by 10 minutes of rest. The validity of the scale as a means of producing different exercise intensities was assessed using a two factor $(\mathrm{RPE} \times$ trial) repeated measures analysis of variance. Intertrial reliability was assessed using intraclass correlation coefficients (ICC) and the bias $\pm 95 \%$ limits of agreement (95\%LoA) procedure. Results-Participants reported no difficulty in using the braille RPE scale. When asked to produce exercise intensities equating to RPE 9, 11, and 13, they elicited mean $\% \mathrm{Vo}_{2} \mathrm{MAx}$ values of $47 \%, 53 \%$, and $63 \%$ respectively. Analysis of variance showed no significant differences in either $\% \mathrm{HR}$ max or $\% \mathrm{Vo}_{2} \mathrm{MAX}$ between trials at each of the three RPEs, but there was a significant difference $(p<0.001)$ in both \%HRMAX and \%Vo MAX between the three RPE levels. All pairwise comparisons of the three different RPEs were significantly different $(p<0.016)$. The ICC between the second and third trial for \%HRMAx was significant $(p<0.05)$ for all three RPEs. Similarly for $\% \mathrm{Vo}_{2} \mathrm{MAx}$, the ICC was significant for RPE 9 and 11 but not 13. The $95 \%$ LoA decreased for both \%HRMAX and $\% \mathrm{Vo}_{2} \mathrm{MAX}$ with each successive trial. Conclusions-Blind participants were successful in using a braille RPE scale to differentiate exercise intensity on a cycle ergometer. In every trial at RPE 13, all participants achieved \%HRMAx and $\% V_{2}$ max levels, which fell within the recommended range for developing cardiorespiratory fitness. Using \%HRMAx as a judge of intertrial reliability, the participants were able to repeat similar exercise intensities after two trials at each of the three RPEs $(9,11,13)$. The same was true for RPE 9 and 11 , when $\% \mathrm{Vo}_{2}$ MAX was used as a judge, but further trials were required to achieve similar reliability at RPE 13. A braille RPE scale can be used by healthy blind people during cycle ergometry, with similar effect to the visual analogue scale recommended for use in healthy sighted people.

(Br F Sports Med 2000;34:297-302)

Keywords: exercise; rating of perceived exertion; braille; blind; cycle ergometry; cardiorespiratory fitness

Borg's ${ }^{1}$ rating of perceived exertion (RPE) scale has become an accepted tool in both the assessment and prescription of exercise. ${ }^{2-4}$ Both collective evaluations and individual studies have recommended that RPE is used as a response measurement (passive estimation) and as an independent variable for regulating exercise intensity (active production) in healthy adults, children, and some clinical populations. $^{15-9}$ To date, no studies have reported the use of RPE in participants who are registered blind, and thus it is yet to be determined whether or not RPE presented in braille is a reliable or valid exercise measurement tool. The nearest application is a study by Abadie,${ }^{10}$ who assessed RPE when the scale was out of view of sighted participants. It would therefore seem prudent that for fitness testing and exercise performed by blind subjects, as in sighted subjects, a measure of RPE should be used to conform to recommended guidelines.

Being blind or vision impaired has been acknowledged as a barrier to physical activity. ${ }^{11}$ In the light of strong epidemiological findings that have linked coronary heart disease and other major illnesses to both low levels of fitness and/or physical inactivity, ${ }^{12}$ the advent and proliferation of modern gymnasia may in part provide blind people with a greater opportunity to become more physically active. Two main educational institutions for the blind in the United Kingdom, at Hereford and Shrewsbury, have successfully used a variety of indoor exercise ergometers within their physical education curricula and extracurricular leisure pursuits programmes. The use of RPE in the sighted has proved to be a practical tool for regulating exercise intensity with a variety of indoor exercise ergometers. ${ }^{13-16}$ The cycle ergometer and the motorised treadmill have been the main modes of activity for assessing the value of RPE. Pioneering studies on the 
reliability and validity of Borg's 6-20 scale were performed by Skinner et $a l,{ }^{17}$ using cycle ergometry, and by Stamford, ${ }^{18}$ using treadmill and step ergometry. Both studies compared RPE responses in estimation mode between progressive and random selections of exercise intensity and showed high correlations upon which to recommend $\mathrm{RPE}$ as a valid and reliable measurement tool. Reliability in both these studies was analysed on a test-retest basis. Lamb et $a l^{19}$ recently performed a similar study using treadmill exercise and reported similar correlations, but questioned the use of the correlation coefficient as a sole determinant of between trial or within participant reliability. They recommended further analysis using limits of agreement (LoA), ${ }^{20} 21$ and on this basis inferred that multiple exposures to the RPE scale of more than two trials were required to achieve a good level of between trial reliability. Whether a similar recommendation is also applicable to the use of RPE in production mode is yet to be confirmed and forms part of the rationale behind this study.

A number of production mode RPE studies have evaluated the reliability of RPE during treadmill exercise, track walking, running, and cycle ergometry. ${ }^{15}{ }^{22-27}$ With the exception of the study by Eston and Williams ${ }^{25}$ the target production mode RPE levels in these studies were determined from estimated responses given during an initial graded maximal exercise test. Byrne and Eston ${ }^{28}$ recommended caution when inferring a target production RPE from graded exercise test estimation mode responses. They reported a mismatch of exercise intensities at a given RPE between estimation and production modes. Eston and Williams ${ }^{25}$ evaluated multiple trial production mode reliability using preselected RPEs during cycle ergometry. Such an application would seem relevant for fieldwork, in which the practicalities and safety issues of administering an initial maximal graded exercise test are alleviated. This study ${ }^{25}$ has not been replicated and yet has been widely cited when RPE reliability is discussed.

In all but one of the studies ${ }^{26}$ cited above, $\mathrm{RPE}$ was recommended as either an independent or adjunctive prescription tool to heart rate, and it was inferred that practice/familiarisation was an important mediator of reliability. The study by Zeni et $a l^{13}$ overtly applied the idea that production mode RPE could be used as an independent intensity regulator. Their study design acknowledged the importance of familiarisation for reliability, and participants performed at least eight exercise sessions on each of the six aerobic exercise machines before the point of beginning data collection on energy expenditure.

In light of there being no previous work on $\mathrm{RPE}$ in blind subjects, the aims of this study were: $(a)$ to assess the validity and reliability of the RPE scale in blind participants by asking them to produce exercise intensities on three separate occasions at three preselected RPEs, during cycle ergometry, using a braille version of Borg's standard 6-20 scale, and (b) to determine whether the heart rate and oxygen uptake

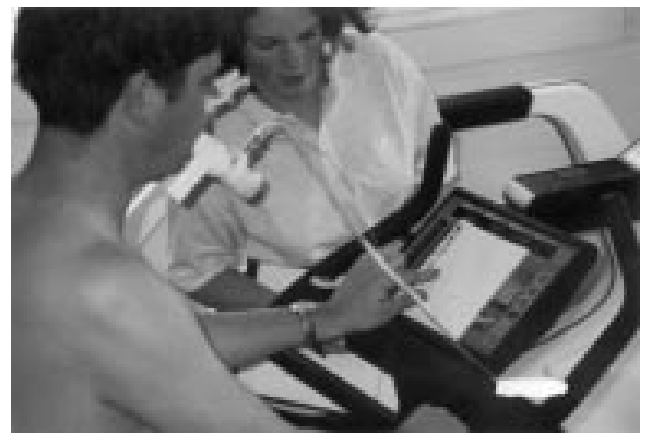

Figure 1 A member of England's blind soccer team participating in the pilot study: measurement of heart rate and expired air with intensity gauged by the braille rating of perceived exertion (RPE) scale mounted on the cycle ergometer's console.

responses of blind participants, for a given produced RPE, were similar to those reported or recommended in recognised guidelines for sighted people. ${ }^{1246}$

\section{Method}

PARTICIPANTS

After approval from the Keele University ethics committee, agreement was then reached with the Royal College for the Blind, Hereford, UK (RCB) to recruit participants. A pilot study was first performed with five members of England's blind soccer team to determine the practicalities of the procedures. The study group consisted of 10 healthy registered blind students of the RCB. There were four women (mean (SD) age 22 (7.6) years) and six men (mean (SD) age 27.3 (11.7) years). All were able to read braille to a high standard. Those with partial vision and/or able to detect light but still registered as officially blind wore special shaded glasses during the exercise tests to ensure that all participants performed the exercise under the same completely unsighted conditions.

\section{EQUIPMENT}

The braille RPE scale was mounted on to the hard flat surface of the cycle ergometer's console (fig 1). The cycle ergometer (Tectrix Bikemax, Irvine, California, USA) operated on an electromagnetically braked system. Work rate was adjustable to increments of $10 \mathrm{~W}$ in the range $10-450 \mathrm{~W}$ and independent of pedal speed. The independence of work rate from pedal speed assumed that participants kept within a recommended pedal speed range of 50-80 rpm. ${ }^{29}$ Furthermore, an "integrated/ overall" RPE has also been shown to be independent of pedal speed in the range 50-80 rpm at a given work rate. ${ }^{30}$ The cycle was fitted with a heart rate receiver linked to a standard two lead wireless chest strap radio transmitter belt (Polar Electro, Oy, Finland). Expired air was measured continuously using a "breathby-breath" system (MedGraphics CPX/D Cardiopulmonary Exercise System; Medical Graphics, Minneapolis, Minnesota, USA), which used a low resistance pneumotach and Pitot tube flow meter. The system was calibrated before each trial for each participant 
Table 1 Group values of $\% H \mathrm{RMAX}_{\mathrm{X}}$ and $\% \mathrm{VO}_{2} \mathrm{MAX}$ for three trials at rating of perceived exertion (RPE) 9, 11, and 13

\begin{tabular}{|c|c|c|c|c|c|c|}
\hline \multirow[b]{2}{*}{$R P E$} & \multicolumn{3}{|l|}{$\% H R M A X$} & \multicolumn{3}{|l|}{$\% V_{O_{2}} M A X$} \\
\hline & Trial 1 & Trial 2 & Trial 3 & Trial 1 & Trial 2 & Trial 3 \\
\hline 9 & $58.2(7.7)$ & $61.3(9.2)$ & $62.8(9.9)$ & $44.5(9.9)$ & $45.9(9.8)$ & $47.5(8.9)$ \\
\hline 11 & $66.3(7.4)$ & $64.4(9.0)$ & $66.3(8.5)$ & $52.7(7.6)$ & $52.5(10.9)$ & $53.2(8.1)$ \\
\hline 13 & $73.6(6.6)$ & $70.9(9.2)$ & $72.8(10.1)$ & $63.5(9.2)$ & $61.8(7.3)$ & $64.7(9.6)$ \\
\hline
\end{tabular}

Values are mean (SD).

HRMAX, maximum heart rate; $\mathrm{VO}_{2} \mathrm{MAX}$, maximum oxygen consumption.

using a 3 litre syringe for volume across a wide range of flow rates and against known gases for the $\mathrm{O}_{2}$ and $\mathrm{CO}_{2}$ analysers.

TESTING PROTOCOL

The following testing protocol was carried out over five days.

\section{Day 1: familiarisation}

The participants gathered in two separate groups to be familiarised with the whole testing procedure. A full description of the testing was explained, including the need for maximal effort, and participants then gave preliminary signed informed consent. Each group was then introduced to Borg's 15 point RPE scale ${ }^{1}$ in its braille format. Participants sat at a desk and were given the opportunity to read over the RPE scale until they stated that they had a clear understanding of both the numerical and verbal statements making up the scale. They were then taken through the recommended and standardised procedure of administration/ familiarisation of the RPE scale, ${ }^{16}$ including: understanding the definition of RPE; "anchoring" the top and bottom ratings to previously experienced sensations of no exertion at all and extremely hard/maximal exertion; explanation of how the scale works; being made aware of giving an "all-over" integrated rating which incorporates both peripheral muscular and central cardiorespiratory sensations; understanding that there was no right or wrong rating; ensuring that the rating represented how hard the participants felt they were working at the time of giving the rating; and having the scale accessible (mounted between the handle bars within finger reach) at all times during testing.

Participants were introduced to wearing the wireless chest strap heart rate radio transmitter belt and practised fitting the mouthpiece and nose clip for the expired air analyses. They practised reading the RPE scale during low intensity cycle ergometry (keeping the pedal speed between 50 and $80 \mathrm{rpm}$ through verbal cues if necessary) while wearing the heart rate monitor, mouthpiece, and nose clip. The pilot study determined that the correct pedal speed range (50-80 rpm) could be easily maintained, through verbal cues from the testing staff, without the use of a metronome. Once all participants had completed this practice session they were then asked to give their final informed consent.

\section{Day 2: graded exercise test}

On the second day, after a five minute low intensity warm up, all participants performed a graded maximal exercise test on the cycle ergometer using standard procedures ${ }^{23}$ to determine maximal oxygen uptake $\left(\mathrm{VO}_{2} \mathrm{MAX}\right)$ and maximal heart rate (HRMAX). Participants kept within the recommended pedal speed range of $50-80 \mathrm{rpm}$.

\section{Days 2-5: production trials}

After a period of at least four hours after the maximal test or on day 3, participants performed their first trial. A trial consisted of three exercise bouts at each of the three preselected RPEs (9, 11, and 13) performed in random order. Each bout consisted of three minutes of low intensity cycling, and then the work rate was adjusted, on command by the participant, to bring the intensity up to the appropriately produced RPE. Expired air and heart rate were measured continuously and recorded when the appropriate RPE and the work rate were unchanged for a period of four minutes, thus establishing steady state measures. Each exercise bout was followed by ten minutes of rest. As with the practice session and the maximal test, participants were thus notified verbally if they went outside of the pedal speed range of 50-80 $\mathrm{rpm}$. The above production trial procedures were repeated twice more on separate days, between days 3 and 5, at the same time of day.

\section{ANALYSIS OF DATA}

The participants' ability to distinctly produce different exercise intensities for a given RPE was assessed using analysis of variance. A two factor repeated measures analysis of variance $(\mathrm{RPE} \times$ trial) was used to test for differences between each of the three RPE levels and between each of the three trials for both $\% \mathrm{VO}_{2} \mathrm{MAX}$ and $\% \mathrm{HRMAX}$. $\alpha$ was set at $\mathrm{p}=0.05$. In cases in which analysis of variance showed a significant difference, a post hoc paired $t$ test, with Bonferoni correction to reduce the possibility of a type I error ( $\alpha$ reduced to $p=0.016)$, was performed to determine the location of pairwise differences.

In accordance with recent recommendations, ${ }^{19}{ }^{21}$ the participants' ability

Table $2 \%$ HRMAX intraclass correlation coefficient (ICC) and the bias $\pm 95 \%$ limits of agreement $( \pm 95 \%$ LoA) for pairwise comparisons across three trials at rating of perceived exertion (RPE) 9, 11, and 13

\begin{tabular}{|c|c|c|c|c|c|c|}
\hline & \multicolumn{2}{|l|}{ RPE 9} & \multicolumn{2}{|l|}{ RPE 11} & \multicolumn{2}{|l|}{ RPE 13} \\
\hline & $T 1-T 2$ & $T 2-T 3$ & $T 1-T 2$ & $T 2-T 3$ & $T 1-T 2$ & $T 2-T 3$ \\
\hline ICC $(95 \% \mathrm{CI})$ & $0.34(-0.24$ to 0.77$)$ & $0.80^{\star}(0.41$ to 0.95$)$ & $0.69^{\star}(0.18$ to 0.91$)$ & $0.88^{\star}(0.61$ to 0.97$)$ & $0.51^{\star}(0.09$ to 0.85$)$ & $0.83^{\star}(0.48$ to 0.95$)$ \\
\hline Bias $( \pm 95 \%$ LoA $)$ & $-8.1(34.1)$ & $-1.5(12.1)$ & $1.9(12.9)$ & $-1.8(8.3)$ & $2.8(15.8)$ & $-1.9(11.2)$ \\
\hline
\end{tabular}

$\mathrm{T} 1-\mathrm{T} 2$, trial $1 v$ trial 2; T2-T3, trial $2 v$ trial 3.

* Statistical significance where the $95 \%$ CI does not include zero.

HRMAX, maximum heart rate. 
Table $3 \% \mathrm{VO}_{2} \mathrm{MAX}$ intraclass correlation coefficient (ICC) and the bias $\pm 95 \%$ limits of agreement ( $\pm 95 \%$ LoA) for pairwise comparisons across three trials at rating of perceived exertion (RPE) 9,11 , and 13

\begin{tabular}{|c|c|c|c|c|c|c|}
\hline & \multicolumn{2}{|l|}{ RPE 9} & \multicolumn{2}{|l|}{ RPE 11} & \multicolumn{2}{|l|}{$R P E 13$} \\
\hline & $T 1-T 2$ & $T 2-T 3$ & $T 1-T 2$ & $T 2-T 3$ & $T 1-T 2$ & $T 2-T 3$ \\
\hline ICC $(95 \% \mathrm{CI})$ & $0.69^{\star}(0.15$ to 0.91$)$ & $0.84^{\star}(0.51$ to 0.96$)$ & $0.46(-0.26$ to 0.84$)$ & $0.78^{\star}(0.33$ to 0.94$)$ & $0.57(-0.06$ to 0.87$)$ & $0.52(-0.08$ to 0.85$)$ \\
\hline Bias $( \pm 95 \%$ LoA $)$ & $-1.4(15.8)$ & $-1.6(10.6)$ & $0.2(20.0)$ & $-0.08(13.2)$ & $1.7(15.7)$ & $-2.9(16.6)$ \\
\hline
\end{tabular}

T1-T2, trial $1 v$ trial 2; T2-T3, trial $2 v$ trial 3.

$\star$ Statistical significance where the $95 \%$ CI does not include zero.

$\mathrm{VO}_{2} \mathrm{MAX}$, maximum oxygen consumption.

to reproduce the same exercise intensity (intertrial reliability) for a given RPE was assessed by the combined use of an intraclass correlation coefficient (ICC; model 2,1) ${ }^{31}$ and the bias $\pm 95 \%$ limits of agreement $(95 \% \mathrm{LoA}) .^{20}$ The ICC and 95\%LoA analysis assessed intertrial agreement for $\% \mathrm{VO}_{2} \mathrm{MAX}$ and $\% \mathrm{HRMAX}$ at each of the three different RPE levels for the following pairwise comparisons: trials 1 and 3 (T1-T3), trials 1 and 2 (T1-T2), and trials 2 and 3 (T2-T3). Statistical significance of the ICC was determined using the $95 \%$ confidence
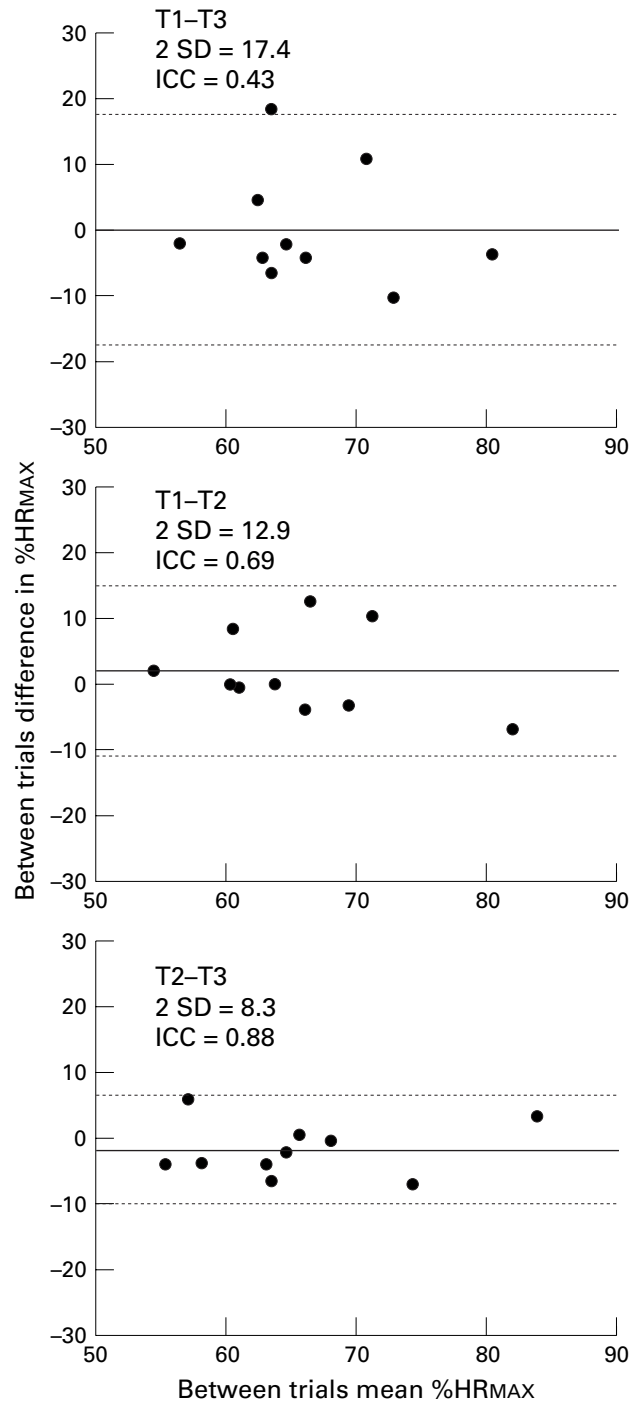

Figure 2 A Bland and Altman ${ }^{20}$ plot illustrating improvement in heart rate reproducibility; bias $\pm 95 \%$ limits of agreement at rating of perceived exertion (RPE) 11 for percentage maximal heart rate (\%HRMAX). T1, T2, and T3, trials 1, 2, and 3; ICC, intraclass correlation coefficient $2,1(n=10)$. interval $(95 \% \mathrm{CI})$. All analyses were performed on SPSS for Windows 8.0.

\section{Results}

The participants reported no difficulty in understanding or using the braille RPE scale. The group's HRMAX and $\mathrm{VO}_{2}$ MAX (mean (SD)) were 184 (5.5) beats/min and 2.2 (0.5) litres/min respectively.

VALIDITY

Table 1 summarises group values of \%HRMAX and $\% \mathrm{VO}_{2} \mathrm{MAX}$ for the three trials at each of the three RPE levels (9, 11, 13). Analysis of variance showed a significant difference in both $\%$ HRMAX $(\mathrm{F}=38.2, \mathrm{p}<0.0005)$ and $\% \mathrm{VO}_{2} \mathrm{MAX}$ $(\mathrm{F}=66.6, \mathrm{p}<0.0005)$ between the three RPE levels. There was no significant interaction between trials and RPE levels for either $\%$ HRMAX $(\mathrm{F}=3.1, \mathrm{p}=0.10)$ or $\% \mathrm{VO}_{2} \operatorname{MAX}(\mathrm{F}$ $=0.58, \mathrm{p}=0.68)$. Post hoc $t$ tests showed that all pairwise comparisons of the three different RPE levels were significantly different $(\mathrm{p}<0.016)$ with respect to both \%HRMAX and $\% \mathrm{VO}_{2} \mathrm{MAX}$.

\section{RELIABILITY}

Analysis of variance disclosed no significant differences across the three trials for either $\%$ HRMAX $\left._{(\mathrm{F}}=0.56, \mathrm{p}=0.48\right)$ or $\% \mathrm{VO}_{2} \mathrm{MAX}(\mathrm{F}$ $=0.33, p=0.72)$ at each of the three RPE levels (table 1). The variability within the $95 \%$ LoA for both $\% \mathrm{HRMAX}$ and $\% \mathrm{VO}_{2} \mathrm{MAX}$ between trials (T1-T3, T1-T2, and T2-T3) decreased with successive trials for all three RPE levels. Tables 2 and 3 summarise the 95\% LoA, and fig 2 provides a graphical example of these data. For \%HRMAx, the ICC increased with successive trials. Statistical significance, as identified by the $95 \% \mathrm{CI}$ excluding zero (tables 2 and 3), was attained for all three RPE levels between T2 and T3. Similar results of T2-T3 for $\% \mathrm{VO}_{2} \mathrm{MAX}$ were true for RPEs 9 and 11 but not for RPE 13, where the ICC (0.52) was not significant $(95 \% \mathrm{CI}-0.08$ to 0.85$)$.

For T2-T3, there was a positive relation between RPE level and the amount of variability in $\% \mathrm{VO}_{2} \mathrm{MAX}$ (table 3 ; ICC and $95 \% \mathrm{LoA}$ ).

\section{Discussion}

The registered blind participants in this study were able to successfully use a braille RPE scale while performing cycle ergometry. They were able to use RPE to differentiate between intensity levels, and improved their ability to repeat a given intensity at the same RPE with each successive trial. Of course, finger reading of the braille scale was facilitated by the stationary position of the upper body during the cycle 
ergometry. It could be recommended that blind participants learn to use and memorise the scale from its braille format during cycle ergometry and then apply the concept during the use of other ergometers. Abadie ${ }^{10}$ found that, when sighted participants who were familiarised with RPE were asked to produce an RPE when the scale was not in view, exertion was underestimated. That study, however, used a treadmill, and it has been reported that using RPE during cycle ergometry provides better reproducibility than treadmill exercise. ${ }^{15}$

DIFFERENTIATING RPE LEVELS IN RELATION TO $\% \mathrm{VO}_{2} \mathrm{MAX}$

Over the three trials, the RPE levels of 9, 11, and 13 equated to mean values of $47 \%, 53 \%$, and $63 \%$ of $\mathrm{VO}_{2} \mathrm{MAX}$ respectively (table 1). These values fell within similar previously reported ranges for cycle ergometry. ${ }^{1425}$ The RPE range $12-16$ is recommended for the development of both health based and rehabilitative cardiorespiratory fitness. ${ }^{2}{ }^{4}$ In keeping with these recommendations, at $\mathrm{RPE} 13$, all our participants in all trials achieved the required exercise intensity range (50-80\% $\mathrm{VO}_{2} \mathrm{MAX}$ ) for developing cardiorespiratory fitness. The $\% \mathrm{VO}_{2} \mathrm{MAX}$ values (table 1 ) were, however, quite varied among the individual participants. At RPE 9, 11, and 13, they were $35-62 \%, 40-65 \%$, and $52-78 \% \mathrm{Vo}_{2}$ MAX respectively. Dunbar et $a l^{2}$ have expressed concern about such between subject variability in the $\mathrm{RPE}-\% \mathrm{VO}_{2} \mathrm{MAX}$ relation, especially with respect to exercise prescriptions for older less well trained people. An important reason for between subject variability at a given RPE is endurance fitness (training status). ${ }^{33}{ }^{34} \mathrm{RPE}$ during cycle ergometry and treadmill exercise were reported as being more strongly related to the lactate or ventilatory threshold than to $\% \mathrm{VO}_{2} \mathrm{MAX}$. The more highly trained subjects exercised at a higher $\% \mathrm{VO}_{2} \mathrm{MAX}$ for a given $\mathrm{RPE}$ than their less trained counterparts. During exercise to music or circuit based exercise, training status appears to be less of a mediator in the $\mathrm{RPE}-\% \mathrm{VO}_{2} \mathrm{MAX}$ relation. ${ }^{35}$ In general, however, it would seem sensible that exercise prescriptions using RPE should account for a subject's training status.

INTERTRIAL RELIABILITY

Our results confirm previous observations ${ }^{25} 36$ that reliability of using RPE improves with more than two trials. The Bland and Altman ${ }^{20}$ plot in fig 2 illustrates how heart rate response becomes less variable with each successive trial. Most of the RPE production mode multiple trial studies have used a motorised treadmill. ${ }^{22-242627}$ Our study adds to the number of studies ${ }^{15} 162536$ that have used the cycle ergometer to evaluate variability and reliability of reproducing a given exercise intensity with RPE. In using heart rate alone to judge the reliability of RPE to reproduce a given exercise intensity, the ICC and 95\%LoA results (tables 2 and 3 and fig 2) showed that, after two trials, RPE provided a high level of reliability. With the same analysis, but using $\mathrm{VO}_{2}$ as the criterion, the reliability argument is somewhat weakened at higher intensities (RPE 13; $\left.>55 \% \mathrm{VO}_{2} \mathrm{MAX}\right)$. Our results therefore highlighted two questions: (a) how well can heart rate on an interday basis be used as a reliable marker of exercise intensity and (b) should heart rate therefore be used as a means of evaluating the reliability of RPE? Interestingly, Dishman $^{5}$ concluded that, within the range $50-70 \% \mathrm{VO}_{2} \mathrm{MAX}$, the reproduction errors in $\mathrm{VO}_{2}$ were no greater when using a target $\mathrm{RPE}$ than when using a target heart rate.

Similar to the results of Lamb et al, ${ }^{19}$ our application of the $95 \%$ LoA analysis (tables 2 and 3, fig 2) showed that between a first and second trial, there was lack of good agreement for many of the individual participants' scores. For example, for $\% \mathrm{VO}_{2} \mathrm{MAX}$ between $\mathrm{T} 1$ and T2, which is a typical test-retest scenario, the 95\%LoA ranges for RPE 9, 11, and 13 were $\pm 15.8 \%, \pm 20 \%$, and $\pm 15.7 \%$ respectively. Unlike Lamb et $a{ }^{19}{ }^{19}$ we performed a third trial analysis (T2-T3), in which agreement further improved at RPE 9 and 11: the $95 \%$ LoA ranges decreased to $\pm 10.6 \%$ and $\pm 13.2 \%$ respectively. These were reductions in variability by a factor of 1.5. Unfortunately, at the higher intensity of RPE 13 (>50\% $\left.\mathrm{VO}_{2} \mathrm{MAX}\right)$, more trials are required to attain such an improvement in intertrial agreement/reliability. In line with the recent available recommendation ${ }^{19}$ for sighted people, blind participants using RPE in braille also require at least two repeated exposures (habituation) to achieve acceptable reliability. It must be acknowledged, however, that Lamb et $a l^{19}$ evaluated RPE in estimation mode using progressive treadmill exercise, whereas our study used two distinctly different variables: steady state RPE in production mode and the use of a cycle ergometer. It is therefore yet to be clearly established whether or not between trials variability and thus reliability are also functions of RPE mode (estimation, production, preferred).

CONCLUSION

These findings show that, when a group of healthy registered blind participants employed a braille RPE scale in production mode to control cycle ergometer intensity, they produced similar cardiorespiratory responses to those outlined in recommended guidelines for healthy sighted people. Furthermore, factors affecting multiple trial reliability are comparable with those in similar studies with sighted subjects. The results highlight that between trials reliability of RPE is a function of the number of trials performed. When RPE reliability is judged by $\% \mathrm{Vo}_{2} \mathrm{MAX}$ and evaluated using a combined analysis of an ICC and limits of agreement, our results show that at least two separate exposures are required in the RPE range 9-12. Attaining exercise intensity reliability for RPE $>13$ using the braille scale in this group of participants requires more than three trials. RPE in braille during cycle ergometery can be used by healthy blind people, with similar effect to the existing exercise testing/ prescription guidelines for healthy sighted people. Future studies should include: a between 
trials reliability comparison of RPE for sighted versus blind; exploration of the number trials required, as judged by $\% \mathrm{VO}_{2} \mathrm{MAX}$, for $\mathrm{RPE}$ to become reliable at higher intensities; determination of the means by which RPE is mediated by the mode (production, estimation, preferred) in which it is used.

We thank Tony Larkin, Director of Physical Education, Roya College for the Blind, Hereford, UK, Gareth Mapp (Lifestyle Fitness, Shrewsbury), Anna Fraser (Exeter University), and Fitness, Shrewsbury), Anna Fraser (Exeter University), and
Penny Smith (Keele University) for their assistance in the exercise assessment trials.

1 Borg GAV. Borg's rating of perceived exertion and pain scales. Champaign, IL: Human Kinetics, 1998.

2 American College of Sports Medicine. Guidelines for exercise testing and prescription, 5th ed. Baltimore: Williams and testing and prescr
Wilkins, 1995.

3 British Association of Sport and Exercise Sciences. Physiological testing guidelines, 3rd ed. Leeds: British Association of Sport and Exercise Sciences, 1997

4 American College of Sports Medicine Position Stand: The recommended quantity and quality of exercise for developing and maintaining cardiorespiratory and muscular strength and flexibility in healthy adults. Med Sci Sports Exerc 1998;30:975-91.

5 Dishman RK. Prescribing exercise intensity for healthy adults using perceived exertion. Med Sci Sports Exerc 1994, 26:1087-94.

6 Noble B, Robertson R. Perceived exertion. Champaign, IL: Human Kinetics, 1996.

7 Williams JG, Eston RG. Exercise intensity regulation. In: Eston RG, Reilly T, eds. Kinanthropometry and exercise physiology laboratory manual: tests, procedures and data. physiology laboratory manual: tests, proced

8 Eston RG, Connolly D. The use of ratings of perceived exertion for exercise prescription in patients receiving exertion for exercise prescription in patien

9 Eston RG, Lamb KL. Effort perception. In: Armstrong N, Van Mechelen W, eds. Oxford textbook of paediatric exercise science and medicine. Oxford: Oxford University Press, 2000:85-91.

10 Abadie BR. Effect of viewing the RPE scale on the ability to make ratings of perceived exertion. Percept Mot Skills 1996, 83:317-18.

11 Hopkins WG, Gaeta H, Thomas AC, et al. Physical fitness of blind and sighted children. Eur 7 Appl Physio 1987;56:69-73.

12 Pate RR, Pratt M, Blair SN, et al. Physical activity and public health: a recommendation from the Centers for Disease Control and Prevention and the American College of Sports Medicine. $¥ A M A$ 1995;273:402-7.

13 Zeni AI, Hoffman MD, Clifford PS. Energy expenditure Zeni AI, Hoffman MD, Clifford PS. Energy expenditure
with indoor exercise machines. $7 A M A$ 1996;275:1424-7.

4 Thomas TR, Ziogas G, Smith T, et al. Physiological and perceived exertion responses to six modes of submaximal perceived exertion responses to six modes
exercise. Res $O$ Exerc Sport 1995;66:239-46.

15 Dunbar CC, Goris C, Michielli DW, et al. Accuracy and reproducibility of an exercise prescription based on ratings of perceived exertion for treadmill and cycle ergomete exercise. Percept Mot Skills 1994;78:1335-44.

16 Dunbar CC, Robertson RJ, Baun R, et al. The validity of regulating exercise intensity by ratings of perceived regulating exercise intensity by ratings

17 Skinner JS, Hutsler R, Bergsteinova V, et al. The validity and reliability of a rating scale of perceived exertion. Med Sci Sports Exerc 1973;5:94-6.

18 Stamford BA. Validity and reliability of subjective ratings of 60

19 Lamb KL, Eston RG, Corns D. The reliability of ratings of perceived exertion during progressive treadmill exercise. $\mathrm{Br}$ perceived exertion during pro
F Sports Med 1999;33:336-9.

20 Bland JM, Altman DG. Statistical methods for assessing agreement between two methods of clinical measurement. Lancet 1986;i:307-10.

21 Nevill AM, Atkinson G. Assessing agreement between measurements recorded on a ratio scale in sports medicine and sports science. Br 7 Sports Med 1997;31:314-18.

22 Smutok MA, Skrinar GS, Pandolf KB. Exercise intensity: subjective regulation by perceived exertion. Arch Phys Med Rehabil 1980;61:569-74.

23 Chow RJ, Wilmore JH. The regulation of exercise intensity by ratings of perceived exertion. $\mathcal{F}$ Cardiac Rehabil 1984;4: 382-97.

24 Eston RG, Davies BL, Williams JG. Use of perceived effort ratings to control exercise intensity in young healthy adults. Eur f Appl Physiol 1987;56:222-4.

25 Eston RG, Williams JG. Reliability of ratings of perceived exertion for regulation of exercise intensity. Brf Sports Med 1988;22:153-4.

26 Whaley MH, Forsyth G. The value of traditional intensity feedback for self regulation of initial exercise training. Fournal of Cardiopulmonary Rehabilitation 1990;10:98-106.

27 Bayles CM, Metz KF, Robertson R, et al. Perceptual regulation of prescribed exercise. Fournal of Cardiopulmonary Rehabilitation 1990;10:25-31.

28 Byrne C, Eston RG. Use of ratings of perceived exertion to regulate exercise intensity: a study using effort estimation and effort production [abstract]. I Sports Sci 1997;16:15.

29 Londeree BR, Moffitt-Gerstenberger J, Padfield JA, et al. Oxygen consumption of cycle ergometry is non-linearly related to work rate and pedal speed. Med Sci Sports Exerc 1997;29:775-80.

30 Marsh AP, Martin PE. Perceived exertion and the preferred cycling cadence. Med Sci Sports Exerc 1998;30:942-8.

31 Rankin G, Stokes M. Reliability of assessment tools in rehabilitation: an illustration of appropriate statistical analyses. Clinical Rehabilitation 1998;12:187-99.

32 Dunbar CC, Stein RA, Goldberg N, et al. Target RPE for post-menopausal women should be based on individual perceptual responses [abstract]. Med Sci Sports Exerc 1999; 31:S83.

33 Travilos AK, Marisi DQ. Perceived exertion during physical exercise among individuals high and low in fitness. Percept Mot Skills 1996;82:419-24.

34 Watt B, Grove R. Perceived exertion; antecedents and applications. Sports Med 1993;15:225-41.

35 Swaine IL, Emmett J, Murty D, et al. Rating of perceived exertion and heart rate relative to ventilatory threshold in women. Br f Sports Med 1995;29:57-60.

36 Eston RG, Campbell L, Lamb KL, et al. Reliability of effort perception for regulating exercise intensity in children: a pilot study using the Cart and Load Effort Rating (CALER) Scale. Pediatric Exercise Science 1999;11:274-5.

\section{Take home message}

The reliability of RPE in braille improves with successive trials. At RPE 13, blind subjects exercise within the recommended intensity limits for developing cardiorespiratory fitness.

\section{Commentary}

Although the health benefits of regular exercise are well established, some sections of our community are faced with more than the usual number of barriers to their participation. Blind people represent a particular case as they are unable to receive the standard visual feedback from the displays on exercise machines. Consequently, whereas a sighted person may be provided with information on their work rate or heart rate, a blind person is deprived of this information. Consequently, the performance of a prescribed exercise intensity becomes problematic. This paper investigates the use of a braille version of the Borg RPE scale for the production of specified exercise intensities in a group of blind subjects. The use of such a scale should facilitate their working at the prescribed exercise intensities and make a small contribution towards assisting this section of our community towards participating in exercise and enjoying the benefits that are available to the fully sighted.

STEVE BIRD

Department of Sport Science, 